\title{
Modic (endplate) changes in the lumbar spine: bone micro-architecture and remodelling
}

\author{
Egon Perilli • Ian H. Parkinson • Le-Hoa Truong • \\ Kuan C. Chong • Nicola L. Fazzalari · \\ Orso L. Osti
}

Received: 2 October 2013/Revised: 1 July 2014/ Accepted: 2 July 2014/Published online: 26 July 2014

(C) The Author(s) 2014. This article is published with open access at Springerlink.com

\begin{abstract}
Purpose In the literature, inter-vertebral MRI signal intensity changes (Modic changes) were associated with corresponding histological observations on endplate biopsies. However, tissue-level studies were limited. No quantitative histomorphometric study on bone biopsies has yet been conducted for Modic changes. The aim of this study was to characterise the bone micro-architectural parameters and bone remodelling indices associated with Modic changes.

Methods Forty patients suffering from disabling low back pain, undergoing elective spinal surgery, and exhibiting Modic changes on MRI (Modic 1, $n=9$; Modic 2, $n=25$; Modic 3, n=6), had a transpedicular vertebral body biopsy taken of subchondral bone. Biopsies were first examined by micro-CT, for 3D morphometric analysis of bone volume fraction (BV/TV), trabecular thickness (Tb.Th), trabecular separation, trabecular number, and
\end{abstract}

\section{E. Perilli $(\bowtie)$}

Medical Device Research Institute, School of Computer Science, Engineering and Mathematics, Flinders University, Sturt Rd.,

Bedford Park, South Australia 5042, Australia

e-mail: egon.perilli@flinders.edu.au

E. Perilli · I. H. Parkinson - L.-H. Truong ·

K. C. Chong · N. L. Fazzalari

Bone and Joint Research Laboratory, SA Pathology and Hanson

Institute, Adelaide, South Australia, Australia

I. H. Parkinson · L.-H. Truong · N. L. Fazzalari

Discipline of Anatomy and Pathology, The University

of Adelaide, Adelaide, South Australia, Australia

K. C. Chong · O. L. Osti

Calvary Healthcare, North Adelaide Campus, 12 Walter St.,

North Adelaide, South Australia 5006, Australia

e-mail: orsolosti@gmail.com structure model index. Then, samples underwent histological analysis, for determination of bone remodelling indices: osteoid surface to bone surface ratio (OS/BS), eroded surface to bone surface (ES/BS) and osteoid surface to eroded surface ratio (OS/ES).

Results Micro-CT analysis revealed significantly higher $\mathrm{BV} / \mathrm{TV}$ (up to $70 \%$ increase, $p<0.01$ ) and Tb.Th (up to $+57 \%, p<0.01)$ in Modic 3 biopsies, compared to Modic 1 and 2. Histological analysis showed significantly lower OS/BS in Modic 2 biopsies (more than $28 \%$ decrease, $p<0.05)$ compared to 1 and 3 . ES/BS progressively decreased from Modic 1 to 2 to 3, whereas OS/ES progressively increased with significantly higher values in Modic 3 (up to $159 \%$ increase, $p<0.05$ ) than in Modic 1 and 2.

Conclusions Significant differences were found in bone micro-architectural parameters and remodelling indices among Modic types. Modic 1 biopsies had evidence of highest bone turnover, possibly due to an inflammatory process; Modic 2 biopsies were consistent with a reduced bone formation/remodelling stage; Modic 3 biopsies suggested a more stable sclerotic phase, with significantly increased BV/TV and Tb.Th compared to Modic 1 and 2, linked to increased bone formation and reduced resorption.

Keywords Modic changes - Vertebra $\cdot$ Micro-computed tomography $\cdot$ Histology $\cdot$ Micro-architecture $\cdot$ Bone remodelling

\section{Introduction}

Low back pain linked to disc degeneration is one among the leading causes of functional incapacitation and chronic disability. Modic et al. [1, 2] in 1988 described and 
classified characteristic magnetic resonance imaging (MRI) changes in the vertebral body bone marrow and endplates, adjacent to degenerating intervertebral discs. Depending on the changes in T1 and T2 MRI signals, three types of lesions were identified (Modic changes): Modic type 1 changes (low $\mathrm{T} 1$ and high $\mathrm{T} 2$ signal) were attributed to increased blood flow within the bone, type 2 changes (high $\mathrm{T} 1$ and $\mathrm{T} 2$ signals) possibly reflecting fatty degeneration of the bone marrow. Type 3 changes (low T1 and T2 signals) were assumed to correlate with subchondral bone sclerosis $[1,2]$. Their occurrence appears to increase with age and is associated with low back pain [3, 4].

Despite the increasing number of studies published in the international literature on Modic changes, their aetiology remains poorly understood [5-9]. Only a few papers have attempted to examine excised vertebral tissue specimens from patients with Modic changes [1-3]. In particular, to the best of the authors' knowledge, no quantitative comparison among the three Modic types, in micro-architectural and remodelling characteristics of the trabecular bone adjacent to the endplates, has yet been published. A quantitative analysis of bone micro-architectural parameters and bone remodelling indices would contribute to the characterisation of Modic changes, important for a better understanding of the pathological process involved.

Thus, the aim of this study is to quantify and compare amongst samples of different Modic types the three-dimensional (3D) bone micro-architecture by means of microcomputed tomography (micro-CT), and bone remodelling characteristics by means of histology, in vertebral bone biopsies taken from adults undergoing spinal surgery and exhibiting Modic changes on preoperative MRI images.

\section{Materials and methods}

Forty patients undergoing lumbar spine surgery with pedicle screw instrumentation and exhibiting Modic changes on preoperative lumbar MRI were selected for this study. In all patients the indication for surgery was disabling low back pain associated with marked disc degeneration at one or two levels. The inclusion criteria for patients were absence of history of bone metabolic diseases, of any anti-osteoporotic treatment, of previous spinal surgery, and of active or suspected ongoing infection. The size of the Modic lesions in terms of area in relation to the vertebra was assessed from the sagittal MRI images [10]. All the 40 lesions accepted for biopsy covered at least $19 \%$ of the area (26\% on average). In those cases where inhomogeneities in the images were present (four cases), the largest homogeneous component in both T1 and T2 weighted signals was taken for classification. All patients were asked to read an information package and to sign an ethics committee approved specific informed consent form prior to surgery. The reading of MRI images and grading of Modic changes was carried out independently and blindly by two radiologists. In case of disagreement, a consensus was negotiated. The inter-observer and intra-observer reliability for the classification of Modic lesions was assessed by means of kappa statistics (Cohen's $\kappa$ ) [11], showing very high agreement $(\kappa=0.83$ and $\kappa=0.92$, respectively).

The age of the 40 patients (17 women, 23 men) was $57 \pm 11$ years (mean \pm standard deviation, SD), age range 35-75 years. The cases were subdivided as follows: Modic $1(n=9)$, Modic $2(n=25)$, Modic $3(n=6)$. At time of surgery, one transpedicular vertebral body subchondral bone biopsy (up to $21 \mathrm{~mm}$ long, $3 \mathrm{~mm}$ in diameter) was harvested under biplanar (anterior-posterior and lateral) image intensifier at the region corresponding to the Modic lesion as seen on preoperative MRI (Fig. 1), using an $8 \mathrm{G}$ Jamshidi needle. The biopsies were always taken at the superior border of the vertebral body, due to the location of the pedicle (Fig. 1). If the lesion as seen on axial MRI was asymmetric, the pedicle on the side of the lesion was chosen, as confirmed by anterior-posterior fluoroscopy. The bone biopsy sample was carefully retrieved from the needle and preserved in $70 \%$ alcohol.

\section{Bone specimen preparation}

All bone samples underwent serial dehydration by increasing the alcohol concentration up to $100 \%$ over a period of 1 week, followed by acetone overnight. No decalcification was done. The biopsies were then infiltrated and embedded in methylmethacrylate [12].

\section{Micro-CT}

A micro-CT scan of the embedded biopsies was carried out to provide a $3 \mathrm{D}$-analysis of the bone micro-architecture using a desktop micro-CT system (SkyScan model 1076,
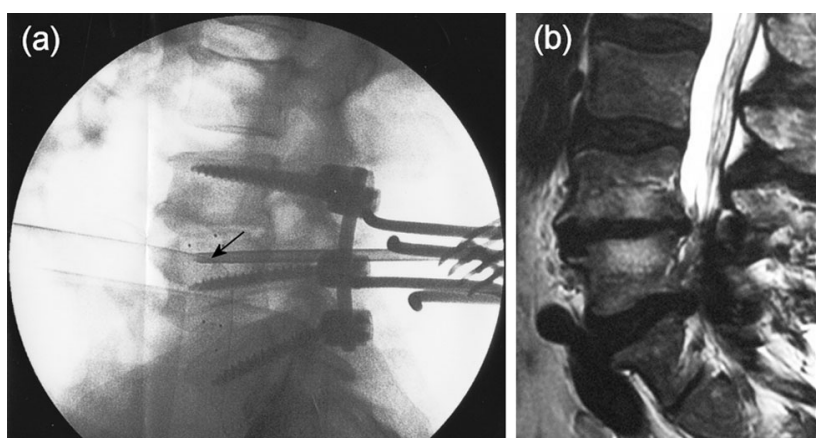

Fig. 1 Intraoperative lateral fluoroscopy image (a), showing position of the biopsy needle (indicated by arrow) within the Modic lesion as seen on preoperative sagittal MRI (b) 
SkyScan NV, Kontich, Belgium) [13, 14]. The sample was centred on the scanner bed and scanned with the following system settings: source voltage $74 \mathrm{kV}$, current $100 \mu \mathrm{A}$, rotation step $0.8^{\circ}$, rotation over $180^{\circ}, 1 \mathrm{~mm}$-thick aluminium filter for beam hardening reduction, isotropic pixel size $8.7 \mu \mathrm{m}$, exposure time $4.7 \mathrm{~s}, 4$ frames averaging. The cross-section images were then reconstructed using a filtered back-projection algorithm (NRecon software, V1.4.4, SkyScan, Kontich, Belgium). Depending on the biopsy, a stack of up to 2,400 cross-section images was reconstructed, with inter-slice distance of 1 pixel $(8.7 \mu \mathrm{m})$, corresponding to a maximum reconstructed length of $21 \mathrm{~mm}$, recreating the full length of the biopsy. The reconstructed axial cross-section images were of $600 \times 600$ pixel each, $8.7 \mu \mathrm{m}$ pixel size, corresponding to images of $5.2 \times 5.2 \mathrm{~mm}$, centred on the bone specimen, and saved in 256 grey-level image file format (.bmp).

For calculation of the micro-architectural parameters, a circular region of interest (ROI) was defined for each crosssection, inscribed in the trabecular bone specimen (software CT Analyser V1.8.0.5, SkyScan, Kontich, Belgium). The diameter of the ROIs $(2.8 \mathrm{~mm})$ was slightly smaller than the bone specimen diameter $(3 \mathrm{~mm})$, to exclude an external annulus of $0.1 \mathrm{~mm}$ thickness, in order to avoid the inclusion of eventual debris due to the cutting procedure. The stack of consecutive ROIs resulted in a cylindrical volume of interest (VOI) of trabecular bone, $2.8 \mathrm{~mm}$ in diameter, up to $21 \mathrm{~mm}$ in height $(10 \pm 4 \mathrm{~mm}$, mean $\pm \mathrm{SD}$ ), over which the calculation of bone volume fraction (BV/TV), trabecular thickness (Tb.Th), trabecular separation (Tb.Sp), trabecular number (Tb.N), and structural model index (SMI), was performed [15, 16]. For the calculation, the cross-section images were segmented (thresholded) into bone and non-bone using a uniform threshold algorithm, with a single threshold value applied to all specimens $[13,17,18]$. The BV/TV (expressed in \%) was calculated as the voxels segmented as bone within the VOI, divided by the voxels constituting the examined VOI, using the marching cubes method $[19,20]$. The Tb.Th (expressed in $\mu \mathrm{m}$ ), a 3D measure of the average thickness of the trabecular bone structure, and the Tb.Sp (expressed in $\mu \mathrm{m}$ ), a 3D measure of the mean distance between the trabeculae, were both computed by using the local spherefitting method [21]. The Tb.N (expressed in $1 / \mathrm{mm}$ ), the number of trabecular plates per unit length $[15,22]$, was calculated using the formula Tb.N $=(\mathrm{BV} / \mathrm{TV}) / \mathrm{Tb}$.Th. The SMI is a topological index, giving an estimate of the characteristic form in terms of plates and rods composing the 3D structure. For ideal plates and rods, this index assumes the values 0 and 3 , respectively, whereas for a mixed structure containing both plates and rods, the SMIvalue lies between 0 and 3, depending on the volume ratio of plates and rods within the structure [23, 25].
Histology

Then, histological sections were prepared for analysis of tissue-level bone remodelling. All embedded bone specimens were trimmed and sectioned with a microtome (Polycut-E, Leica SP 2600, Leica Microsystems, Wetzlar, Germany). Sections, $5 \mu \mathrm{m}$ thick, were stained using the von Kossa silver method and counterstained with hematoxylin and eosin (H\&E) to distinguish between the mineralized bone, the cellular components of the marrow, and the osteoid. Histomorphometric measurements were done over three sections taken through the biopsy, in order to be representative of the whole biopsy, using an optical microscope (Leica DFC 480) with an ocular-mounted $10 \times 10$ graticule at a magnification of $\times 100$. The histological measurements for quantifying the remodelling parameters were: percent osteoid surface to bone surface (OS/BS, expressed in \%); percent eroded surface to bone surface (ES/BS, expressed in \%); osteoid surface to eroded surface ratio (OS/ES) [12, 24].

\section{Statistical analysis}

The values of age, trabecular micro-architectural parameters and bone remodelling parameters showed a normal distribution within each group (Shapiro-Wilks test, $p>0.05$ for all). To compare the values of age, trabecular micro-architectural parameters and bone remodelling parameters between the three Modic groups, a Fisher's protected least significant difference (post hoc) test was performed (SAS software, SAS Inc, Cary, NC, USA). In the literature, in the 'normal' ageing population (non-pathological), a significant decrease of trabecular bone volume fraction with increasing age is reported. To investigate this dependency in samples with Modic changes in the present study, a linear regression analysis was done. For all the statistical tests, significance level test was set at $p=0.05$.

\section{Results}

All the bone samples were successfully examined using micro-CT and histology (Table 1). There were no significant differences in age amongst the three Modic groups ( $p=0.72$, Fisher's PLSD test). A 3D-micro-CT representation of three biopsies corresponding to three Modic types is shown in Fig. 2.

\section{Micro-CT}

Micro-CT analysis revealed significantly higher BV/TV in Modic type $3(+70 \%, \mathrm{p}<0.01$, and $+56 \%, \mathrm{p}<0.01$, respectively) compared to Modic type 1 and type 2 (Table 1, Fig. 3). Similarly, Tb.Th was significantly higher 
Table 1 Descriptive data of age, 3D micro-architectural parameters from micro-CT and remodelling indices from histology, in the vertebral bone biopsies of the three Modic types

\begin{tabular}{lccc}
\hline & Modic $1(n=9)$ & Modic $2(n=25)$ & Modic 3 $(n=6)$ \\
\hline $\begin{array}{l}\text { Age (years) } \\
\text { Bone micro-architecture }\end{array}$ & $55 \pm 12(37,71)$ & $57 \pm 11(35,75)$ & $60 \pm 14(45,75)$ \\
BV/TV $(\%)$ & $16.3 \pm 6.1(8.5,24.4)$ & $17.8 \pm 5.9(8.4,28.8)$ & $27.7 \pm 6.6(19.7,33.8)$ \\
Tb.Th $(\mu \mathrm{m})$ & $153 \pm 41(108,236)$ & $155 \pm 40(98,268)$ & $240 \pm 56(165,298)$ \\
Tb.Sp $(\mu \mathrm{m})$ & $591 \pm 67(507,701)$ & $622 \pm 113(383,809)$ & $606 \pm 73(525,687)$ \\
Tb.N $(1 / \mathrm{mm})$ & $1.0 \pm 0.3(0.7,1.5)$ & $1.2 \pm 0.3(0.7,1.9)$ & $1.2 \pm 0.3(0.8,1.6)$ \\
SMI & $1.2 \pm 1.2(-1.9,2.0)$ & $1.0 \pm 0.7(-1.1,2.0)$ & $0.8 \pm 1.2(-1.1,1.8)$ \\
Bone remodelling & & & $13.3 \pm 5.1(6.8,24.9)$ \\
OS/BS $(\%)$ & $18.5 \pm 6.8(6.1,28.6)$ & $8.2 \pm 4.3(1.7,22.0)$ & $19.2 \pm 5.2(11.3,26.9)$ \\
ES/BS $(\%)$ & $10.6 \pm 3.0(6.1,15.1)$ & $2.4 \pm 2.1(0.6,7.4)$ & $5.6 \pm 3.5(2.1,11.7)$ \\
OS/ES & $1.7 \pm 0.4(1.0,2.4)$ & $4.4 \pm 2.0(1.7,7.5)$ \\
\hline
\end{tabular}

Bone micro-architectural parameters: bone volume fraction, BV/TV (\%); trabecular thickness, Tb.Th $(\mu \mathrm{m})$; trabecular separation $(\mu \mathrm{m})$; trabecular number, Tb.N (1/mm); structure model index, SMI

Bone remodelling indices (static indices of bone turrnover): osteoid surface to bone surface ratio, OS/BS (\%); eroded surface to bone surface ratio, ES/BS (\%); osteoid surface to eroded surface ratio, OS/ES

Values indicated as mean $\pm \mathrm{SD}(\min , \max )$

Modic $1 \quad$ Modic $2 \quad$ Modic 3
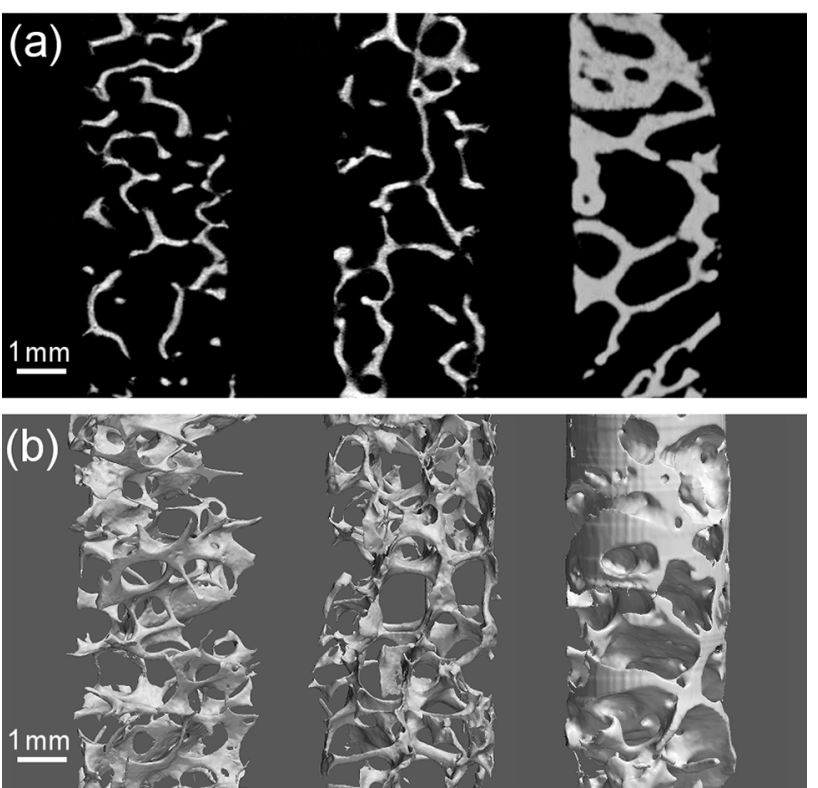

Fig. 2 Vertebral bone biopsies from the three Modic types examined by micro-CT $(8.7 \mu \mathrm{m} /$ pixel $)$ : a $2 \mathrm{D}$ micro-CT cross-section images and $\mathbf{b}$ 3D micro-CT rendering

in Modic type $3(+57 \%, \mathrm{p}<0.01$, and $+55 \%, \mathrm{p}<0.01$, respectively) compared to Modic type 1 and type 2 (Table 1, Fig. 3). Tb.Sp, Tb.N and SMI were not significantly different between groups $(p>0.05$ for all the parameters), although SMI values showed a consistent trend to decrease from Modic type 1 towards type 3 (Table 1).
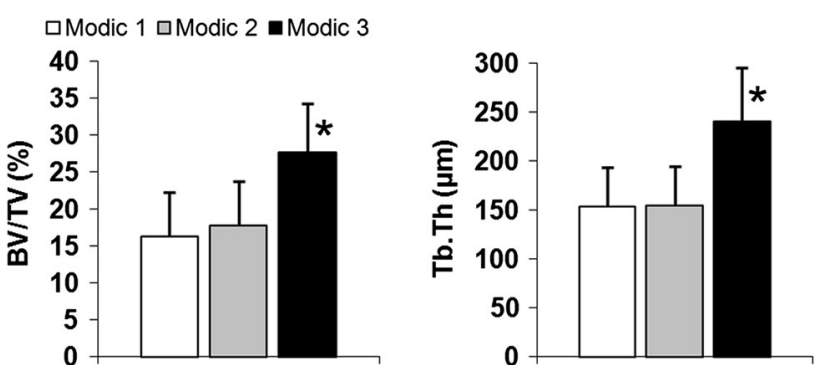

Fig. 3 3D micro-CT analysis, bone micro-architectural parameters. Bar diagram of average value and standard deviation (error bars) of parameters reported in Table 1, with statistical significance for comparison (Fisher's PLSD post hoc test): BV/TV and Tb.Th were significantly increased in Modic 3 specimens, compared to Modic 1 and $2(* p<0.01$ for both parameters, in both comparisons). Bone volume fraction, BV/TV $(\%)$; trabecular thickness, Tb.Th $(\mu \mathrm{m})$

For the regression 'BV/TV vs. age', the data of the three groups were pooled together. There was no significant relationship between $\mathrm{BV} / \mathrm{TV}$ and age $\left(R^{2}=0.04, p=0.24\right)$.

Histology

Histological analysis showed reduced OS/BS ratio in Modic $2(-28 \%, \mathrm{p}<0.05$, and $-31 \%, \mathrm{p}<0.05$, respectively) compared to both Modic 1 and Modic 3 (Table 1, Fig. 4). The ES/BS ratio progressively decreased from Modic 1 to 2 to 3 , with statistically significant lower values in Modic $3(-47 \%, \mathrm{p}<0.05)$ compared to Modic 1). The OS/ES ratio increased from Modic 1 to Modic 2 and 3 , with significantly higher values in Modic 3 $(+159 \%, \mathrm{p}<0.05$, and $+83 \%, \mathrm{p}<0.05$, respectively) compared to both Modic 1 and Modic 2 ( Fig. 4). 

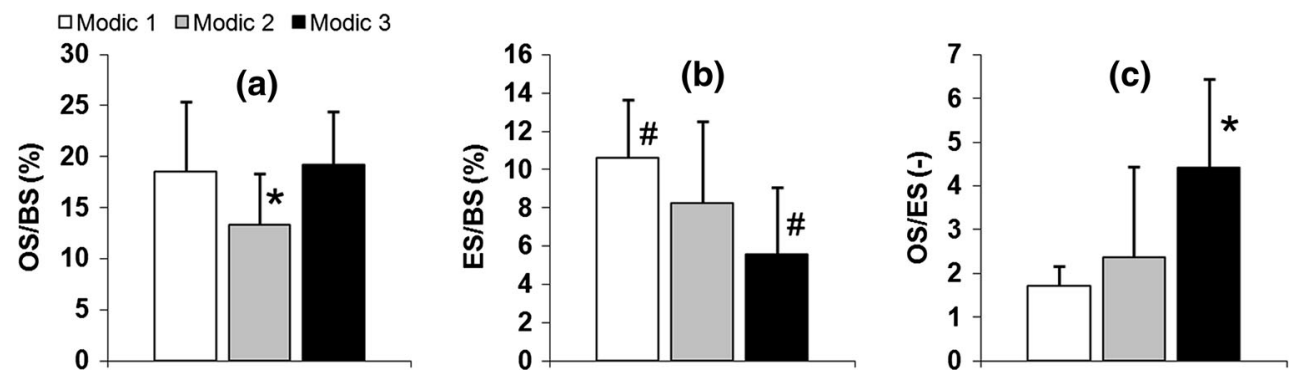

Fig. 4 Bone histological analysis (von Kossa and H\&E staining), remodelling indices. Bar diagram of average value and standard deviation (error bars) of parameters reported in Table 1, with statistical significance for comparison (Fisher's PLSD post hoc test): a in Modic 2 the OS/BS ratio was significantly decreased compared to Modic 1 and 3 (*p $<0.05$ in both comparisons). b Significantly

\section{Discussion}

The findings of this study show significant differences in 3D bone micro-architectural parameters as measured by micro-CT and in bone remodelling indices as measured by histology among Modic types. It can be postulated, as suggested by Modic et al. [1,2] that different Modic types represent different stages of the same pathological process, linked to the adjacent disc.

Significant increases in BV/TV and Tb.Th were found in Modic 3 compared to Modic 1 and 2, whereas the other investigated micro-architectural parameters, Tb.Sp, Tb.N, and SMI were not statistically different amongst the three Modic types. These results suggest that the increase in BV/ TV seen in Modic type 3 was mainly due to thickening of the trabeculae. For SMI a non-significant decrease was found, which is a trend towards a more plate-like structure in Modic 3, compared to Modic 1 and 2 [23, 25]. This lack of statistical significance could be simply due to the small number of Modic 3 samples.

Histological analysis showed significant increase in the bone formation to bone erosion ratio (OS/ES) in Modic 3 samples, compared to Modic 1 and 2. This finding is consistent with both reduced bone resorption in Modic 3 samples compared to Modic 1, indicated by the significantly decreased ES/BS, as well as with the increased bone formation, with OS/BS significantly higher in both Modic 3 and in Modic 1 compared to 2.

Taken together, Modic 1 and 2 biopsies differed mainly in bone remodelling indices. On the other hand, bone samples in Modic 3 showed significant differences in both micro-architecture and also remodelling indices, compared to the other two Modic types. Modic 3 changes were consistent with a more stable sclerotic phase of the process, showing significantly higher BV/TV and thicker trabeculae compared to Modic 1 and 2, with reduced bone resorption and increased bone formation activity. reduced ES/BS ratio in Modic 3 compared to Modic $1\left({ }^{\#} p<0.05\right)$. c Significantly higher OS/ES ratio in Modic 3 compared to Modic 1 and $2(* p<0.05$ in both comparisons). Osteoid surface to bone surface ratio, OS/BS (\%); eroded surface to bone surface ratio, ES/BS (\%); osteoid surface to eroded surface ratio, OS/ES

In our series, no obvious difference was observed in the overall size of different types of Modic changes as seen on MRI. Specimens were taken of same length due to the standard size of the biopsy needle (8G Jamshidi needle), with the biopsy always contained within the Modic lesions as confirmed by fluoroscopy.

Modic et al. [1] in 1988 postulated that the different Modic types may represent different stages of the same pathological process, linked to the adjacent disc, reflecting a spectrum of marrow changes: Modic 1 changes indicating an ongoing degenerative process, disruption and fissuring of the endplates, with marrow replacement by small blood vessels; Modic 2 indicating an ongoing degenerative process, disruption of endplates, with bone marrow replaced by yellow marrow (fat); and Modic 3 changes reflecting bone sclerosis seen on plain radiographs, an indication of woven bone in the vertebral body, rather than a reflection of the marrow element.

The present study done on excised bone biopsies, examining bone micro-architecture parameters and remodelling indices, supports the hypotheses of different pathological stages postulated by Modic et al.. Modic 1 associated changes appeared to be consistent with a more active phase of the pathology, showing high bone formation ratio and erosion ratio. Modic 2 biopsies were more suggestive of a reduced bone formation/remodelling stage, as indicated by the remodelling indices. Modic 3 changes were consistent with the bone sclerosis assumed by Modic et al. with significantly higher BV/TV and thicker trabeculae, and high bone formation/erosion ratio, as well as reduced resorption. This might also explain the characteristic appearance of Modic type 3 in MRI images (low T1 and low T2 signals), which is likely a reflection of decreased or absent marrow and/or fat in these MRI images, due to the increased amount of bone in Modic 3 as found by micro-CT in this study, indicated by the increased BV/TV (up to $+70 \%$ ) and Tb.Th (up to $+57 \%$ ). 
In a previous study, Kuisma et al. [34] compared clinical MRI and clinical CT images taken from patients exhibiting Modic changes. Their study showed that in Modic type 3, the low T1 and T2 signals seen in MRI, corresponded to endplate bone sclerosis as indicated in the CT images, with increased Hounsfield units (HU) (increased bone mineral density), which is in agreement with our results using micro-CT. It is worth mentioning that the spatial resolution of clinical CT, as used in their study (slice thickness $1.25 \mathrm{~mm}$ and $0.625 \mathrm{~mm}$ interval, in-plane resolution not indicated) enables measurement of mineralized tissue in terms of HU, but does not allow characterization of the trabecular bone micro-architecture [26], in particular in the vertebrae, where the trabeculae can be as thin as $80 \mu \mathrm{m}$ [27]. In other words, it does not allow to identify whether the changes in HU, were due to more, or thicker, trabeculae, or same number of trabeculae but more mineralized, or a combination of the above. Moreover, no bone biopsies were taken in that study, to assess the bone micro-architecture and bone remodelling. Micro-CT is a relatively recent, non-destructive high-resolution imaging technique, validated by $2 \mathrm{D}$ histology and physical 3D phantoms [18, $25,26,28$, capable of $2 \mathrm{D}$ and 3D quantitative characterisation of excised bone segments at a detection level not achievable with conventional MRI or CT scanners. It has a spatial resolution (10 $\mu \mathrm{m}$ range), which is $10-100$ times better than conventional clinical CT or MRI scanners, and can accurately differentiate between the trabecular and cortical bone compartments and non-osseous tissues, as opposed to conventional clinical CT and MRI imaging [14, 26, 27, 29]. Although it has been used in spine studies previously, it has never been used to study in Modic changes before [30-32]. It is typically used for ex vivo scanning of excised human and animal bones [14, 29] or in vivo scanning of small animals, at micrometer resolution [33]. For clinical CT scans on human patients in vivo, the improvement in spatial resolution (decrease of pixel size) is currently limited by the corresponding increase in radiation dose [28]. However, within the restrictions of the radiation dose, it can be expected that thanks to technological developments and improvements in spatial resolution, clinical CT systems might be increasingly used in future for studying Modic changes in vivo, as done by Kuisma et al. [34]. Recently, pre-clinical High-Resolution peripheral Quantitative CT (HR-pQCT) systems have become available, for in vivo scanning of peripheral parts of the human body, such as the wrist and ankle [35] with a pixel size $(82 \mu \mathrm{m})$ approximating that of micro-CT.

In a previous study, Modic et al. examined excised endplate tissue using histological analysis, however, the analysis was only qualitative (descriptive), not quantitative, limited to Modic type 1 and type 2 patients with only three patients per group, and with no Modic type 3 patients [1].
In the present study, excised bone biopsies from 40 patients were examined, with all the three Modic groups represented. To the best of the author's knowledge, this is the first report on bone micro-architecture and remodelling indices in Modic changes done on excised bone biopsies. Furthermore, the specimens were subjected to micro-CT analysis, which enabled visualisation and quantification of the bone micro-architecture in 3D, as well as to histology, which enabled an assessment of the remodelling indices. This represents both the novelty as also the strength of this study.

A limitation of this study was the sample size (40 patients in total), in particular for the occurrence in the Modic 3 group (6 patients), compared to the Modic 1 group (9 patients) and Modic 2 groups (25 patients). The patients examined were those eligible for this study undergoing surgery at the hospital and exhibiting Modic changes on preoperative MRI, over a 24 months period. Despite the sample size, significant differences between the groups in micro-architecture and remodelling indices were detected. Moreover, the overall prevalence rate of patients in each group was found to be consistent with that reported in the literature, with Modic 2 being the most common followed by Modic 1, whereas Modic 3 appears to be the least common [3, 36, 37]. Some studies even reported no Modic type 3 patients in their series [7, 38].

In longitudinal studies reported in the literature, spontaneous conversions of Modic types have been found within a time span of 1-year, with Modic 1 to 2 being he most common, including conversions from type 2 to 1 , and from type 1 to normal [3]. Modic type 1 represents the major transition point from normality, and as such, appears to be a main focus of research [3]. The exact mechanism causing Modic changes is still not clear; it could be a response to early inflammatory stages of the degenerating disc [9]. In the present study, Modic 1 samples had the highest bone turnover with increased OS/BS and ES/BS, possibly due to an inflammatory reaction increasing both osteoblastic and osteoclastic activity. A systematic review of the literature and of mechanisms of conversion between Modic changes was done by Zhang et al. [3]. According to current literature, the two major causes for the pathogenesis of Modic changes are biomechanical and biochemical [3]. With regards to the biomechanical cause, micro-fractures and fissures in the endplates have been reported, in relation to human disc degeneration [1]. As such, Modic changes might be due to altered mechanical stress, resulting in histological changes, leading to signal intensity changes on MRI, namely the Modic changes. Biochemical causes on the other hand, imply that Modic changes may result from an inflammatory reaction to toxic mediators or infection from the degenerating disc [39]. An intervertebral disc herniation could be the entry point of bacteria into the disc, and the Modic changes could be therefore the result of 
anaerobic bacteria leading to oedema and inflammation surrounding the extruded intra-vertebral nuclear material $[9,40]$. A recent study has suggested antibiotic treatment as an effective option for a specific subgroup of patients with Modic type 1 changes [9]. Significant controversy has followed, with a number of authors raising concerns about the methodology and conclusion of that study [41-52]. Within the restrictions of our specimen size, and our study being a bone micro-architecture and remodelling study, not aimed at investigating bacterial infection, our histological slides of the bone biopsies (H\&E stained) did not show associated oedema and no cellular infiltrate to indicate overt inflammation. However, the material analysed in the present study included bone biopsies only, and not intervertebral disc which may contain bacteria and show inflammation. This remains a controversial topic, in need of further research as suggested by numerous letters to this journal [41-52]. Hence, evidence supporting the biomechanical and biochemical hypotheses is still not sufficient, and it remains unclear how they may play a synergistic role in the development of Modic changes in the human body [3].

The trabecular BV/TV in this study was not significantly related to the age of the patients $\left(R^{2}=0.04, p=0.24\right.$, age range 35-75 years). This finding in Modic changes is different from that for normal (non-pathological) vertebral bone reported in the literature, where, in bone extracted from similar anatomical locations within the vertebrae and examined with histology and micro-CT, the trabecular BV/ TV shows significant decrease with age (e.g., $R^{2}=0.61$, $p<0.05$, age range 23-95 years; or $R^{2}=0.61, p<0.05$, age range 57-98 years) $[30,53]$. The absence of correlation with age in Modic changes in our study is a novel finding, and is likely linked to the increased remodelling activity found in the bone. It is worth noting that this result is similar to observations in bones of patients with osteoarthritis (e.g., in the proximal femur), for which typical age-related changes found in normal patients are altered, showing no age-dependent decrease in BV/TV $[17,54,55]$. We acknowledge that having a comparison with specimens from a control group exhibiting no Modic changes in our study may have strengthened our findings.

In conclusion, this is the first quantitative study done on excised bone biopsies, examining quantitative bone histomorphometric changes among the three different Modic types, using micro-CT and histology. The findings have shown significant differences in bone micro-architectural parameters and bone remodelling indices among Modic types. This suggests that different Modic types might represent different stages of the same pathological process, linked to the adjacent disc. Modic 1 specimens have highest bone turnover, with increased OS/BS and ES/BS, possibly due to an inflammatory reaction; Modic 2 changes are consistent with reduced bone formation/ remodelling; Modic 3 changes suggest a more stable sclerotic phase, with significantly increased bone volume fraction and trabecular thickness when compared to Modic 1 and 2, linked to increased bone formation and reduced bone resorption. Our study has not revealed any specific active pathological condition behind Modic changes, which therefore, at this point in time, should be interpreted as being merely a reactive response to the degenerating intervertebral disc.

Acknowledgments Dr KC Chong was supported by an educational grant from DePuy Johnson and Johnson Australia.

\section{Conflict of interest None.}

Open Access This article is distributed under the terms of the Creative Commons Attribution License which permits any use, distribution, and reproduction in any medium, provided the original author(s) and the source are credited.

\section{References}

1. Modic MT, Masaryk TJ, Ross JS, Carter JR (1988) Imaging of degenerative disk disease. Radiology 168:177-186

2. Modic MT, Steinberg PM, Ross JS, Masaryk TJ, Carter JR (1988) Degenerative disk disease: assessment of changes in vertebral body marrow with MR imaging. Radiology 166:193-199

3. Zhang YH, Zhao CQ, Jiang LS, Chen XD, Dai LY (2008) Modic changes: a systematic review of the literature. Eur Spine J 17:1289-1299

4. Wang Y, Videman T, Battie MC (2012) Modic changes: prevalence, distribution patterns, and association with age in white men. Spine J 12:411-416

5. Karchevsky M, Schweitzer ME, Carrino JA, Zoga A, Montgomery D, Parker L (2005) Reactive endplate marrow changes: a systematic morphologic and epidemiologic evaluation. Skeletal Radiol 34:125-129

6. Kaapa E, Luoma K, Pitkaniemi J, Kerttula L, Gronblad M (2012) Correlation of size and type of Modic types 1 and 2 lesions with clinical symptoms: a descriptive study in a subgroup of patients with chronic low back pain on the basis of a university hospital patient sample. Spine 37:134-139 (Phila Pa 1976)

7. Kuisma M, Karppinen J, Niinimaki J, Ojala R, Haapea M, Heliovaara M, Korpelainen R, Taimela S, Natri A, Tervonen O (2007) Modic changes in endplates of lumbar vertebral bodies: prevalence and association with low back and sciatic pain among middle-aged male workers. Spine 32:1116-1122 (Phila Pa 1976)

8. Hutton MJ, Bayer JH, Powell JM (2011) Modic vertebral body changes: the natural history as assessed by consecutive magnetic resonance imaging. Spine 36:2304-2307 (Phila Pa 1976)

9. Albert HB, Sorensen JS, Christensen BS, Manniche C (2013) Antibiotic treatment in patients with chronic low back pain and vertebral bone edema (Modic type 1 changes): a double-blind randomized clinical controlled trial of efficacy. Eur Spine $\mathbf{J}$ 22:697-707

10. Luoma K, Vehmas T, Gronblad M, Kerttula L, Kaapa E (2008) MRI follow-up of subchondral signal abnormalities in a selected group of chronic low back pain patients. Eur Spine J 17:1300-1308

11. Fleiss JL (1981) Statistical methods for rates and proportions. Wiley, NY 
12. Tsangari H, Findlay DM, Fazzalari NL (2007) Structural and remodelling indices in the cancellous bone of the proximal femur across adulthood. Bone 40:211-217

13. Perilli E, Briggs AM, Kantor S, Codrington J, Wark JD, Parkinson IH, Fazzalari NL (2012) Failure strength of human vertebrae: prediction using bone mineral density measured by DXA and bone volume by micro-CT. Bone 50:1416-1425

14. Perilli E, Parkinson IH, Reynolds KJ (2012) Micro-CT examination of human bone: from biopsies towards the entire organ. Ann Ist Super Sanita 48:75-82

15. Parfitt AM, Drezner MK, Glorieux FH, Kanis JA, Malluche H, Meunier PJ, Ott SM, Recker RR (1987) Bone histomorphometry: standardization of nomenclature, symbols, and units. Report of the ASBMR Histomorphometry nomenclature committee. J Bone Miner Res 2:595-610

16. Bouxsein ML, Boyd SK, Christiansen BA, Guldberg RE, Jepsen KJ, Müller R (2010) Guidelines for assessment of bone microstructure in rodents using micro-computed tomography. J Bone Miner Res 25:1468-1486

17. Perilli E, Baleani M, Ohman C, Baruffaldi F, Viceconti M (2007) Structural parameters and mechanical strength of cancellous bone in the femoral head in osteoarthritis do not depend on age. Bone 41:760-768

18. Perilli E, Baruffaldi F, Visentin M, Bordini B, Traina F, Cappello A, Viceconti M (2007) MicroCT examination of human bone specimens: effects of polymethylmethacrylate embedding on structural parameters. J Microsc 225:192-200

19. Lane NE, Thompson JM, Haupt D, Kimmel DB, Modin G, Kinney JH (1998) Acute changes in trabecular bone connectivity and osteoclast activity in the ovariectomized rat in vivo. J Bone Miner Res 13:229-236

20. Lorensen WE, Cline HE (1987) Marching cubes: a high resolution 3D surface construction algorithm. Comput Graph 21:163-169

21. Hildebrand T, Rüegsegger P (1997) A new method for the modelindependent assessment of thickness in three-dimensional images. J Microsc 185:67-75

22. Parfitt AM, Mathews CH, Villanueva AR, Kleerekoper M, Frame B, Rao DS (1983) Relationships between surface, volume, and thickness of iliac trabecular bone in aging and in osteoporosis. Implications for the microanatomic and cellular mechanisms of bone loss. J Clin Invest 72:1396-1409

23. Hildebrand T, Rüegsegger P (1997) Quantification of bone micro architecture with the structure model index. Comput Methods Biomech Biomed Engin 1:15-23

24. Tsangari H, Findlay DM, Zannettino AC, Pan B, Kuliwaba JS, Fazzalari NL (2006) Evidence for reduced bone formation surface relative to bone resorption surface in female femoral fragility fracture patients. Bone 39:1226-1235

25. Perilli E, Baruffaldi F, Bisi MC, Cristofolini L, Cappello A (2006) A physical phantom for the calibration of three-dimensional X-ray microtomography examination. J Microsc 222:124-134

26. Müller R (2009) Hierarchical microimaging of bone structure and function. Nat Rev Rheumatol 5:373-381

27. Hildebrand T, Laib A, Müller R, Dequeker J, Rüegsegger $P$ (1999) Direct three-dimensional morphometric analysis of human cancellous bone: microstructural data from spine, femur, iliac crest, and calcaneus. J Bone Miner Res 14:1167-1174

28. Müller R, Hahn M, Vogel M, Delling G, Rüegsegger P (1996) Morphometric analysis of noninvasively assessed bone biopsies: comparison of high-resolution computed tomography and histologic sections. Bone 18:215-220

29. Müller R, Van Campenhout H, Van Damme B, Van Der Perre G, Dequeker J, Hildebrand T, Ruegsegger P (1998) Morphometric analysis of human bone biopsies: a quantitative structural comparison of histological sections and micro-computed tomography. Bone 23:59-66

30. Chen H, Shoumura S, Emura S, Bunai Y (2008) Regional variations of vertebral trabecular bone microstructure with age and gender. Osteoporos Int 19:1473-1483

31. Kim DG, Hunt CA, Zauel R, Fyhrie DP, Yeni YN (2007) The effect of regional variations of the trabecular bone properties on the compressive strength of human vertebral bodies. Ann Biomed Eng 35:1907-1913

32. Briggs AM, Perilli E, Parkinson IH, Wrigley TV, Fazzalari NL, Kantor S, Wark JD (2010) Novel assessment of subregional bone mineral density using DXA and pQCT and subregional microarchitecture using micro-CT in whole human vertebrae: applications, methods, and correspondence between technologies. J Clin Densitom 13:161-174

33. Perilli E, Le V, Ma B, Salmon P, Reynolds K, Fazzalari NL (2010) Detecting early bone changes using in vivo micro-CT in ovariectomized, zoledronic acid-treated, and sham-operated rats. Osteoporos Int 21:1371-1382

34. Kuisma M, Karppinen J, Haapea M, Lammentausta E, Niinimaki J, Tervonen O (2009) Modic changes in vertebral endplates: a comparison of MR imaging and multislice CT. Skeletal Radiol 38:141-147

35. Boutroy S, Bouxsein ML, Munoz F, Delmas PD (2005) In vivo assessment of trabecular bone micro architecture by high-resolution peripheral quantitative computed tomography. J Clin Endocrinol Metab 90:6508-6515

36. Peterson CK, Humphreys BK, Pringle TC (2007) Prevalence of Modic degenerative marrow changes in the cervical spine. J Manipulative Physiol Ther 30:5-10

37. Albert HB, Manniche C (2007) Modic changes following lumbar disc herniation. Eur Spine J 16:977-982

38. Weishaupt D, Zanetti M, Hodler J, Min K, Fuchs B, Pfirrmann CW, Boos N (2001) Painful lumbar disk derangement: relevance of endplate abnormalities at MR imaging. Radiology 218:420-427

39. Braithwaite I, White J, Saifuddin A, Renton P, Taylor BA (1998) Vertebral end-plate (Modic) changes on lumbar spine MRI: correlation with pain reproduction at lumbar discography. Eur Spine J 7:363-368

40. Albert HB, Kjaer P, Jensen TS, Sorensen JS, Bendix T, Manniche C (2008) Modic changes, possible causes and relation to low back pain. Med Hypotheses 70:361-368

41. O'Dowd J, Casey A (2013) Antibiotics a cure for back pain, a false dawn or a new era? Eur Spine J 22:1694-1697

42. Dean BJ (2013) Do these results apply to the 'intervention naive' patient? Eur Spine J 22:1702

43. Sotto A, Dupeyron A (2013) Letter to the editor concerning: "Antibiotic treatment in patients with chronic low back pain and vertebral bone edema (Modic type 1 changes): a double-blind randomized controlled trial of efficacy" by Albert $\mathrm{HB}$ et al. Eur Spine J (2013) 22:697-707. Eur Spine J 22:1704-1705

44. Dean BJ, Davies BM (2013) No conflict of interest? Eur Spine J 22:1700

45. Shubhakaran K, Khichar RJ (2013) Backache and infection. Eur Spine J 22:2348

46. Ahmad Z, Rai A, Donell S, Crawford R (2013) Letter to the editor concerning "Antibiotic treatment in patients with chronic low back pain and vertebral bone edema (Modic type 1 changes): a double-blind randomized controlled trial of efficacy" by Albert HB et al. Eur Spine J (2013) 22:697-707. Eur Spine J

47. Albert HB (2013) Answer to the editorial of j. O'Dowd and a Casey. Eur Spine J 22:1698-1699

48. Albert HB (2013) Answer to the Letter to the Editor of K. Shubhakaran et al. entitled "Backache and infection" concerning "Antibiotic treatment in patients with chronic low back pain and 
vertebral bone edema (Modic type 1 changes): a double-blind randomized controlled trial of efficacy" by Albert H.B. et al., Eur Spine J (2013) 22:697-707. Eur Spine J

49. Albert HB (2013) Answer to the Letter to the Editor of Benjamin John Floyd Dean entitled "Do these results apply to the 'intervention naive' patient?" concerning "Antibiotic treatment in patients with chronic low back pain and vertebral bone edema (Modic type 1 changes): a double-blind randomized controlled trial of efficacy" by Albert HB et al., Eur Spine J (2013) 22:697707. Eur Spine J 22:1703

50. Albert HB (2013) Answer to the Letter to the Editor of Benjamin John Floyd Dean et al. entitled "No conflict of interest?" concerning "Antibiotic treatment in patients with chronic low back pain and vertebral bone edema (Modic type 1 changes): a doubleblind randomized controlled trial of efficacy" by Albert HB et al., Eur Spine J (2013) 22:697-707. Eur Spine J 22:1701

51. Albert HB (2013) Answer to the letter to the editor of A. Sotto et al. concerning "Antibiotic treatment in patients with chronic low back pain and vertebral bone edema (Modic type 1 changes): a double-blind randomized controlled trial of efficacy" by Albert HB et al., Eur Spine J (2013) 22:697-707. Eur Spine J 22:1706

52. Lambert P, Albert HB (2013) Answer to the Letter to the Editor of Zafar Ahmad et al. concerning "Antibiotic treatment in patients with chronic low back pain and vertebral bone edema (Modic type 1 changes): a double-blind randomized controlled trial of efficacy" by Albert HB et al., Eur Spine J (2013) 22:697707. Eur Spine J

53. Thomsen JS, Ebbesen EN, Mosekilde L (2002) Zone-dependent changes in human vertebral trabecular bone: clinical implications. Bone 30:664-669

54. Crane GJ, Fazzalari NL, Parkinson IH, Vernon-Roberts B (1990) Age-related changes in femoral trabecular bone in arthrosis. Acta Orthop Scand 61:421-426

55. Jordan GR, Loveridge N, Power J, Clarke MT, Reeve J (2003) Increased cancellous bone in the femoral neck of patients with coxarthrosis (hip osteoarthritis): a positive remodelling imbalance favoring bone formation. Osteoporos Int 14:160-165 